\title{
Téoros
}

Revue de recherche en tourisme

\section{Entretien avec Michael BARRETT, directeur associé, Service des ressources renouvelables, de l'environnement, du territoire et des parcs, Administration régionale Kativik (ARK)}

\author{
Alain A. Grenier
}

Volume 31, numéro 1, 2012

URI : https://id.erudit.org/iderudit/1020720ar

DOI : https://doi.org/10.7202/1020720ar

Aller au sommaire du numéro

Éditeur(s)

Université du Québec à Montréal

ISSN

0712-8657 (imprimé)

1923-2705 (numérique)

Découvrir la revue

Citer ce document

Grenier, A. A. (2012). Entretien avec Michael BARRETT, directeur associé, Service des ressources renouvelables, de l'environnement, du territoire et des parcs, Administration régionale Kativik (ARK). Téoros, 31(1), 132-133.

https://doi.org/10.7202/1020720ar 


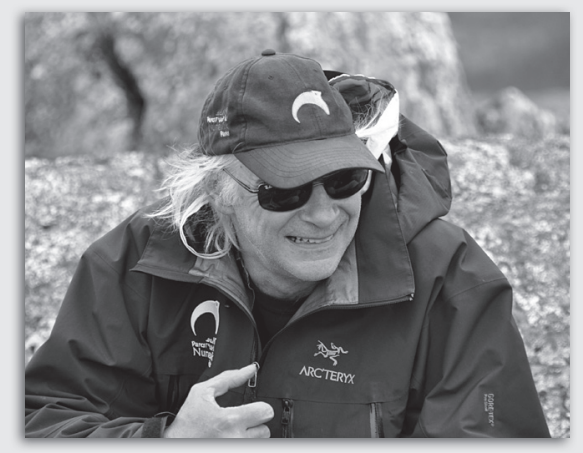

\title{
Entretien
}

\author{
avec Michael BARRETT, directeur associé, \\ Service des ressources renouvelables, de \\ I'environnement, du territoire et des parcs, \\ Administration régionale Kativik (ARK)
}

Propos recueillis par Alain A. Grenier, directeur et rédacteur en chef de Téoros

\begin{abstract}
Comment devient-on directeur associé du Service des ressources renouvelables, de l'environnement, du territoire et des parcs?

On recule loin. Je suis originaire du nord de l'Ontario. J'étais gardien de forêt l'été et universitaire l'hiver. J'ai obtenu un diplôme en anthropologie. J'ai ensuite commencé à travailler avec les Cris et les pourvoyeurs à la Baie-James, au début des années 1970. En 1974, j'ai commencé à travailler à Kuujjuaq avec les Inuits, durant six mois. Je travaillais alors pour le ministère des Affaires indiennes du gouvernement canadien.

Pour tout dire, je suis quelqu'un qui a l'expérience du travail en plein air. Avec la nouvelle Administration régionale Kativik, on m'a d'abord chargé du programme d'aide aux chasseurs. L'objectif de ce programme était d'amener un soutien (financier) aux activités de prélèvement (chasse et pêche) dont une partie de la cueillette revient aux communautés. Ce programme comprenait plusieurs activités dont le congélateur municipal, qui consiste toujours, en gros, à aider les chasseurs à défrayer certains coûts liés à la chasse, dans le Grand Nord, en retour d'un partage de la viande obtenue avec le village. En effet, tous les Inuits ne sont pas en mesure de chasser. Ce programme permet alors de maintenir les activités traditionnelles et les valeurs associées au partage, très importantes dans les communautés du Nord. II y avait donc beaucoup à faire avant que n'arrive la question des parcs.
\end{abstract}

\section{Quand vous a-t-on convié à travailler sur le projet de parcs au Nunavik?}

Mon travail m'avait amené à bien connaître les 14 communautés du territoire. C'est ainsi que, en 1996, on m'a demandé de vérifier le dossier du premier parc national, Pingualuit. Une entente complémentaire de la Convention de la Baie-James disait que le Québec devait établir un parc au cratère du Nouveau-Québec, aujourd'hui appelé Pingualuit. Comme généraliste, on m'a demandé de travailler sur le projet pour voir à l'établissement du parc.

La demande de créer le parc des Pingualuit vient de la communauté de Kangiqsujuaq, qui a beaucoup d'imagination. Une petite communauté de 400-450 personnes qui veut protéger un cratère, c'est remarquable. Les Inuits de cette communauté sont derrière l'entente Raglan. Cette entente, conclue en 1995 entre la Société Minière Raglan du Québec, la Société Makivik, les communautés de Salluit et de Kangiqsujuaq, ainsi qu'avec les corporations foncières de Salluit et de Kangiqsujuaq, visait particulièrement les retombées du projet minier. Les Inuits voulaient protéger Pingualuit et, du même coup, s'assurer de bénéficier de l'activité économique, par le biais d'emplois et de formation de la main-d'œuvre. C'était une première au Canada. C'était une façon imaginative d'assurer qu'il y ait des retombées positives pour le Nunavik. Aujourd'hui, les Inuits vont plus loin. Ils veulent à présent nettoyer plusieurs sites d'exploration minière abandonnés.

Notre travail, au Service des ressources renouvelables, de l'environnement, du territoire et des parcs, c'est de faire la gestion de tout cela. Nous sommes en quelque sorte le pendant nordique de la Sépaq, qui gère la plupart des parcs nationaux du reste du Québec.

Les collaborateurs de ce numéro ont largement abordé le processus de création des parcs au Nunavik. Quel a été le rôle de l'Administration régionale Kativik?

En gros, dans les années 1990, nous avons établi un plan directeur pour la gestion du territoire. On y a identifié les zones essentielles pour les activités de prélèvement (chasse et pêche) et qui demeurent importantes pour les communautés. Les Inuits ont alors indiqué les endroits qu'ils voulaient protéger pour la conservation. Leurs propositions de parcs coïncidaient parfaitement avec la nécessité de protéger certains espaces du développement industriel.

Initialement, les parcs étaient créés pour protéger des échantillons représentatifs des écosystèmes d'un État. Dans quelle mesure cela est-il toujours vrai aujourd'hui par rapport au rôle du parc comme stimulant économique au Nunavik?

La création des parcs se veut une façon de protéger les territoires du développement industriel. En retour, les parcs ont le potentiel de soutenir une activité économique. C'est d'autant plus important pour des villages de souvent quelques centaines de personnes. On a plusieurs pourvoyeurs qui montent dans le Nord pour les activités de chasse, traditionnelles ou sportives, pour les touristes à la recherche d'une expérience en milieu naturel. Alors les parcs constituent un attrait majeur. Les parcs soutiennent aussi en partie les communautés par leur centre d'interprétation, situé dans les villages, mais aussi par les emplois des gardiens de parcs.

Certains de vos parcs peuvent atteindre des superficies de plus de 5000 km². Quels sont les impacts de la géographie particulière du Nord et des distances qu'elle impose aux visiteurs de ces parcs?

La direction des parcs contrôle l'accès au territoire car, si on a trop de monde, cela peut créer des problèmes de conservation. On accepte l'avion ou l'hélicoptère l'été pour les visiteurs, mais on ne peut se poser dans le parc sans l'approbation de la direction. L'hiver, on recommande le ski ou le traîneau à chiens. Comme les parcs sont souvent situés à plusieurs kilomètres des villages, la motoneige est utilisée par les employés dans le cadre de leur travail et par les visiteurs comme moyen d'accès au parc. Un Inuit, par contre, peut aller partout avec la motoneige. 


\section{Cette situation peut-elle engendrer des conflits avec les touristes?}

Cela fait partie du règlement de la Baie-James. Les Inuits ont le droit de se déplacer et le droit de prélèvement partout. Et le visiteur doit accepter ces règles. Les Inuits agissent à titre de guides. Alors ils expliquent ces règles aux touristes, aux centres d'interprétation ou durant l'activité. Le comité d'harmonisation et la direction des parcs s'occupent de gérer tout conflit potentiel, notamment quant aux activités touristiques qui pourraient gêner la chasse et la pêche traditionnelles.

Le comité d'harmonisation est un lieu d'échange entre les gestionnaires des parcs et les communautés. II a pour rôle de se réunir au moins deux fois par année pour échanger sur le développement des parcs, les difficultés rencontrées et les solutions. Sa composition varie d'un parc à l'autre, mais il comporte des représentants des corporations foncières locales et des conseils municipaux, du gouvernement du Québec, de l'ARK et de la Société Makivik.

Si on n'établit pas de parcs pour la conservation, ça va être l'exploitation ou l'exploration minière partout. C'est donc primordial d'établir un équilibre en identifiant et en créant des zones de protection de la biodiversité avant le démarrage des mines. N'oublions pas que les parcs jouent aussi un rôle d'éducation.

\section{Parlons de cette mission d'éducation et de vos visiteurs. Qu'en est-il ?}

Fait surprenant : on a moins de visiteurs en provenance du Sud et davantage de visiteurs originaires du Nord. Nous avons beaucoup de jeunes Inuits qui viennent avec leur école. Pour eux, la nature fait partie de leur patrimoine. Toutefois, comme les Inuits vivent aujourd'hui dans des villages, et que les gens travaillent dans les mines ou dans les services, alors les familles sortent moins, et c'est normal aussi.

Le parc favorise donc le séjour en milieu naturel. C'est l'occasion pour les Inuits, et les jeunes en particulier, de sortir de leur village. Ils peuvent voir la nature, leur nature, dans un contexte différent de la chasse et de la pêche. Avec leurs professeurs, avec les guides, ils étudient la flore, la faune et la beauté des sites. On a eu un groupe de $5^{\mathrm{e}}$ secondaire qui nous a dit que, pour eux, le séjour dans le parc, ici, au Nunavik, avait été la plus belle expérience de leur vie. Cela a été une surprise pour nous de voir que les parcs peuvent avoir un impact si positif pour ces jeunes.

\section{Les Inuits vivent donc avec la sédentarisation un détachement de la nature, un peu comme nous le vivons depuis l'époque de la Révolution industrielle? \\ Oui, exactement. Ce n'est pas tout le monde, bien sûr, mais pour nous ce fut une révélation.}

\section{Vous avez mentionné plus tôt que le parc pouvait être créateur d'emplois pour les Inuits. Qu'en est-il? \\ Dans nos services, qui comprennent la protection de la faune, près de $80 \%$ des employés sont Inuits de langue maternelle. Par exemple, chaque directeur de parc est Inuit. On a trois gardiens dans chacun des deux parcs actuels qui sont aussi Inuits. II en sera de même au troisième parc qui s'en vient. Nous avons deux technicien- nes en administration qui sont Inuites. II y a aussi des Inuits dans d'autres fonctions.}

La conservation des espaces naturels et la gestion des activités humaines qui y sont permises n'est pas chose facile, nulle part

\section{sur la planète. Le Nord - et en particulier le Grand Nord québécois - présente-il des défis particuliers?}

Oui, il y en a plusieurs. En premier lieu, le développement du réseau des parcs lui-même est un défi. En 2004 et 2009, les parcs nationaux des Pingualuit et Kuururjuaq ont été créés et, dans les prochains mois, le parc national Tursujuq sera créé. Nous sommes à développer un modèle de gestion qui pourra ensuite être appliqué aux autres parcs du réseau. On espère établir deux autres parcs, Ulittaniujalik en 2013 et Baie-aux-Feuilles (Tasiujaq) en 2015. Après cela, il y a la construction des infrastructures. On ne parle pas ici de grandes infrastructures mais du minimum pour accueillir les visiteurs, comme les centres d'accueil et d'interprétation et les camps aménagés. On pourra ensuite travailler à l'accueil des visiteurs et au marketing.

Au-delà de ces questions, un de nos grands défis est la gestion des distances : la logistique du transport des visiteurs vers les parcs mais aussi de l'approvisionnement en matériaux, le déplacement des équipes d'entretien, etc. De juin à octobre, on peut accéder au parc par avion. Donc il faut bien prévoir : l'entrée du matériel l'hiver, par motoneige, et la construction l'été. II y a aussi toute la question de la sécurité des visiteurs avec les distances importantes des communautés. Dans certains parcs, on a des ours polaires qui viennent l'été, dans les monts Torngat, notamment. Cela soulève des approches particulières.

$\|$ y a aussi le recrutement et la formation du personnel. On y travaille déjà. C'est difficile car il nous faut trouver du personnel qualifié. Cependant, cela est très intéressant car nous allons avoir des Cris, des Naskapis et des Inuits qui agiront comme gardiens de parcs. C'est donc une opération multiculturelle et c'est un défi fort intéressant. II faut dire que, lorsque nous avons commencé, personne n'avait d'expérience en matière de parc, ici, dans le Nord. On a fait appel à des biologistes et des généralistes pour faire l'inventaire du terrain. On a ensuite embauché une personne qui avait de l'expérience dans les parcs du Sud. Malgré tout, nous étions devant une page blanche. Nous avions comme principe de protéger les territoires des communautés dans un but de conservation. Nous avons beaucoup de soutien du gouvernement du Québec. Cela nous a permis de développer un modèle complètement différent, adapté au Nord. Tout n'est pas terminé. On y travaille encore.

Graduellement, nous avons fait appel à différents experts pour former le personnel dans différentes sphères : l'accueil touristique, la gestion d'activités sportives comme le kayak, la descente de rivière, etc.

II y a aussi toute la question du développement durable et écologique. Ce n'est pas facile de faire des activités écologiques, à nos latitudes. L'électricité ici est produite par des génératrices alimentées au pétrole. Dans les parcs, on essaie de fonctionner le plus possible avec des panneaux solaires. On essaie de faire le maximum pour l'environnement mais, pour entrer ici, on doit venir par avion. Tout le matériel que l'on a ici vient par avion. À cause des distances, on n'a pas le choix; sinon, il n'y aurait pas de visiteur. Et nos communautés en ont besoin.

\section{La question du transport est donc primordiale. Croyez-vous qu'on puisse arriver dans un avenir relativement proche à des transports à la fois moins polluants et moins coûteux entre le Sud et le Nord québécois?}

Ça va arriver, mais comment? Est-ce que cela se fera par le chemin de fer? Si on a des mines, on va avoir un chemin de fer. Mais sans mine... Les mines n'ont pas que des aspects négatifs, vous savez. Elles aident au développement du Nord et des communautés. En retour, le développement des parcs vient contrebalancer le développement minier. 